\title{
AI TECHNOLOGIES AS A FACTOR OF COMPETITIVENESS OF A BUSINESS ENTITY AT THE PRESENT STAGE OF HUMAN DEVELOPMENT
}

\author{
Dmitry Alferyev ${ }^{1}$, Ekaterina Khusainova ${ }^{2}$ \\ ${ }^{1}$ Federal state budgetary institution of science, Vologda research center of the Russian Academy of Sciences \\ ${ }^{2}$ Kazan State Power Engineering University, str. Krasnoselskaya, 51, 420066, Kazan, Russia
}

\begin{abstract}
AI technologies are the main tool in the formation of management decisions at the present stage of human society development. An economic entity's possession of a more advanced, functionally verified, and effective such intellectual tool is its exclusive competitive advantage in the current market environment. In this regard, the author attempts to justify the promotion of AI technologies at the state level. If this initiative is implemented, this country will gain a foothold or reach the level of a developed state in the entire set of participating subjects of the international arena.
\end{abstract}

\section{Introduction}

Global digitalization of the global socio-economic space causes the emergence and development of new methods for studying and managing various processes and phenomena. One of the leading methods in this regard has become AI technology, the emergence of which is due to the thorough development of computer technologies. This tool opens up new opportunities for humanity in any field of activity where it will be applied, and therefore the purpose of these theses is to justify the promotion of this tool at the state level in order to gain competitive advantages in the world hierarchy.

The advantage of AI technologies is the ability to solve poorly formalized problems that have a dynamic nature of the processes occurring in them. However, we note that problems in their classical sense can also be solved using AI technologies and the answers can be obtained as close to optimal, or even better than those found using classical methods of forecasting, analysis and data processing. The simulated artificial neural networks (the structure of the mathematical model obtained using AI technologies) make it possible to establish a relationship in the generated model between the resulting estimates and other structural elements without specifying the type of relationship. In this regard, artificial intelligence technology can be considered as a universal approximation method [1].

In 2018, AI technologies were actively discussed at the world economic forum and were in fact one of the main topics of this event. Many of its participants pointed out that the possession of advanced AI technology can radically change the balance of power in the political and international arena, namely, have a positive impact on the level of socio-economic development of underdeveloped countries.
At this event, the head of Sberbank of Russia Gref G. O. pointed out that artificial intelligence is a disruptive technology of the XXI century. Its owner will be able to gain a serious competitive advantage in the field of activity where this technology will be implemented, and leave behind their competitors, even if they were previously leaders in their field of activity [2].

Major international corporations, primarily related to the development of computer software, have become actively engaged in developing the potential of AI technologies. Their representatives are such well-known companies as Microsoft, Amazon, Google, Facebook and IBM [3]. The result of this Alliance is an Association that currently conducts joint research projects in the field of AI applications (development of intelligent search engines, analysis and research of clients, development of methods for optimizing and improving the efficiency of artificial neural networks,...).

In addition to large companies, medium-sized and small businesses have become significantly more active, which, due to the variety of market niches that have been formed, have identified the areas in which they will work, and have actively begun to develop promising sales markets for their future products [4]. Examples of such organizations include Nervana, which develops computer chips for Intel. In accordance with new technological trends, their chips are equipped with a built-in AI, which allows, in accordance with observations about the operation of the carrier, to optimize its further activity, which in turn affects its wear resistance and speed. A similar commercial project is currently being implemented by the startup Cerebras, located in the US Silicon valley. According to Forbes, by the beginning of 2018, he managed to attract investment capital in the amount of $\$ 100$ million. 
The Chinese People's Republic has taken an active position in the development of Autonomous intelligent systems. Two Beijing companies: Horizon Robotics and Cambricon, as well as their American counterparts, are developing computer chips that support AI technologies and are actively funded by the Chinese government in this regard. China's interest in artificial intelligence is also confirmed by the Ministry of science and technology, which currently obliges Chinese companies dealing with issues and problems in this area to develop competitive chips that are not inferior to those produced by the American company Nvidia.

These chips find their application in another promising area of the future industry, related to the automation of robotic technology and mechatronics. This industry is the automotive industry. In it, you can observe the introduction of autopilots installed on vehicles of transport and road traffic (Tesla, Toyota, Uber,...). Robotic devices that simulate complex physical processes of living organisms (Boston Dynamics, iRobot, KUKA Roboter,...) also play an important role in this path.

An example of successful implementation of AI technologies in terms of economic efficiency is the introduction of deep learning systems developed by DeepMind for Google data centers, which allowed increasing the energy efficiency of server and network equipment located there by about $15 \%$ [5].

According to analysts of the international company Gartner, by 2020 , about $40 \%$ of the available technologies will be based on the application and use of artificial neural network algorithms. A similar opinion is held by another large analytical company MarketsandMarkets [5]. Its experts note that in the period from 2016 to 2022, the highest growth rates of this market are expected, the average annual value of which will be about $65.3 \%$.

As noted above, the possibilities of using AI technologies are not limited to this [6-9]. At the moment, it has also been actively implemented in the field of health and medicine, replacing doctors when making a diagnosis and prescribing therapy based on collected clinical data and chemical and biological markers. At the same time, the error rate of such a program is lower than that of most specialists in this field. AI is used in the context of predictive Analytics, predicting future trends and trends, and based on them, build appropriate decision measures to accelerate the implementation of positive effects or eliminate negative ones in the future.

When implementing AI technologies, there are a number of problems that, however, in our opinion, are not so disastrous in comparison with the effects that will be achieved if they are implemented.

A serious barrier to implementing intelligent software is the reluctance of individuals to recognize the benefits of using AI, which they explain by a number of problems. One of them is the fear of the possibilities and advantages of a Superintelligent program, which in theory should surpass the human mind in all its aspects, but when considering this problem in more detail, it can be noted that the leap in the development of artificial intelligence technologies is primarily due to the computing capabilities of modern machines. And although they successfully cope with individual tasks, they still do it as an imitation, based on the search for possible combinatorial solutions and the speed of this search. In this regard, modern intelligent systems are primarily aimed at replacing standard, identical, unified works and can be used in various fields of activity, such as sorting objects (commodity products, documentation), extrapolating trends and trends from past data to study the dynamics of statistical indicators. However, it should be noted that these operations can be implemented if there is input data. Without them, the learning process of such a program is impossible.

Another problem is the disappearance of some professions and, as a result, the unemployment of a significant segment of the population, but this situation was also inherent when handicraft manual labor was replaced by machine labor. At that time, some professions disappeared, but this happened gradually and was accompanied by the time needed to calibrate and optimize the new technology. In addition, there are new jobs associated with the maintenance of new devices. At present, as in the past, there is a significant need for specialists involved in programming and computer technology, as well as for personnel who will design new modern smart machines. In fact, many professionals who are directly involved in the creative process will continue to engage in the activities that they performed before the advent of AI technologies, regardless of what field of knowledge they belong to, be it biology, chemistry, law or agriculture. What will change is that they will be more able to engage in creative work and, with the help of modern artificial intelligence technology, be able to get to the truths that they knew how to get, but could not do it because of the physical capabilities and conditions available at that time.

Thus, it can be noted that AI technologies are currently being actively used by the world's leading and developed economies, as well as large corporations in various areas, which indicates their clear advantage over the tools and tools that were used earlier. This, in turn, prescribes their use for smaller entities as well.

It is also worth noting that there are certain barriers that hinder the spread of this technology due to fears of negative consequences from its use. But by and large they are similar to the provisions that were promoted by the Luddites during the transition from manual to manufactured labor.

\section{References}

1. D.A. Alferyev, AI Technologies as a method of predictive Analytics, Artificial society, 4 (2018) URL: https://artsoc.jes.su/s207751800000137-9-1

2. A.A. Urunov, The Influence of artificial intelligence, Internet technology on the labor market and the stratification of society // step into the future: artificial intelligence and the digital economy: proceedings of the 1 st International scientific-practical conference. Vol. 4. State 
University of management. M.: Publishing house of goo, 220-225 (2017)

3. R. Parloff, From 2016: Why Deep Learning Is Suddenly Changing Your Life, Fortune (2016). URL: https://fortune.com/longform/ai-artificialintelligence-deep-machine-learning/

4. S. Manukov, Startup boom where you didn't guess // Expert Online. URL: http://expert.ru/2018/01/16/startapovskij-bum-tamgde-ne-zhdali/

5. L.A. Tsvetkova, Artificial intelligence Technologies as a factor of digitalization of the economy of Russia and the world, Economics of science, 3(2), 126-144 (2017).

6. A. Ross, Industry of the future, 351 (2017).

7. E. Siegel, To Calculate the future. Who will click, buy, lie or die (Publisher: Alpina publisher, 2017)

8. K. Schwab, Technologies of the fourth industrial revolution, 320, (Eksmo, 2018).

9. K. Schwab, The fourth industrial revolution, 288, (Publisher: Alpina publisher, 2018)

10. D. Mazhitov, M. Ermilova, E. Altukhova, T. Maksimova, and O. Zhdanova, Development of Technologies and Processes in Environmental Management, E3S Web of Conferences 135, 04045 (2019). doi:10.1051/e3sconf/201913504045.

11. A.G. Polyakova, E. M. Akhmetshin, A. V. Pavlyuk, and G. V. Meshkova, Investment Appeal of a Region and its Impact on Investment Inflows, Entrepreneurship and Sustainability Issues 7 (2), 1089-1097 (2019) . doi:10.9770/jesi.2019.7.2(21) 